\title{
Short-term ankle motor performance with ankle robotics training in chronic hemiparetic stroke
}

\author{
Anindo Roy, PhD; ${ }^{\text {* }}$ Larry W. Forrester, PhD; ${ }^{1}$ Richard F. Macko, MD $^{1-2}$ \\ ${ }^{1}$ Departments of Neurology and Physical Therapy and Rehabilitation Sciences, University of Maryland School of Med- \\ icine, Baltimore, MD; and Maryland Exercise and Robotics Center of Excellence, Rehabilitation Research and Devel- \\ opment Service, Baltimore Department of Veterans Affairs Medical Center (VAMC), Baltimore, MD; ${ }^{2}$ Department of \\ Medicine, University of Maryland School of Medicine, Baltimore, MD; and Geriatric Research, Education, and Clini- \\ cal Center, Baltimore VAMC, Baltimore, MD
}

\begin{abstract}
Cerebrovascular accident (stroke) often results in impaired motor control and persistent weakness that may lead to chronic disability, including deficits in gait and balance function. Finding ways to restore motor control may help reduce these deficits; however, little is known regarding the capacity or temporal profile of short-term motor adaptations and learning at the hemiparetic ankle. Our objective was to determine the shortterm effects of a single session of impedance-controlled ankle robot ("anklebot") training on paretic ankle motor control in chronic stroke. This was a double-arm pilot study on a convenience sample of participants with chronic stroke $(n=7)$ who had residual hemiparetic deficits and an equal number of ageand sex-matched nondisabled control subjects. Training consisted of participants in each group playing a target-based video game with the anklebot for an hour, for a total of 560 movement repetitions in dorsiflexion/plantar flexion ranges followed by retest 48 hours later. Task difficulty was adjusted to ankle range of motion, with robotic assistance decreased incrementally across training. Assessments included robotic measures of ankle motor control on unassisted trials before and after training and at 48 hours after training. Following exposure to the task, subjects with stroke improved paretic ankle motor control across a single training session as indexed by increased targeting accuracy (21.6 +/- 8.0 to $31.4+/-4.8, p=0.05$ ), higher angular speeds (mean: $4.7+/-1.5$ degrees/s to $6.5+/-2.6$ degrees/s, $p<0.01$, peak: $42.8+/-9.0$ degrees/s to $45.6+/-9.4$ degrees/s, $p=0.03$ ), and smoother movements (normalized jerk: $654.1+/-103.3 \mathrm{~s}^{-2}$ to $537.6+/-86.7 \mathrm{~s}^{-2}, p<0.005$, number of speed peaks: $27.1+$ / -5.8 to $23.7+/-4.1, p<0.01)$. In contrast, nondisabled subjects did not make statistically significant gains in any metric after training except in the number of successful passages (32.3 +/-
\end{abstract}

7.5 to $36.5+/-6.4, p=0.006)$. Gains in all five motor control metrics were retained $(p>0.05)$ at 48 hours in both groups. Robust maintenance of motor adaptation in the robot-trained paretic ankle over 48 hours may be indicative of short-term motor learning. Our initial results suggest that the anklebot may be a flexible motor learning platform with the potential to detect rapid changes in ankle motor performance poststroke.

Key words: ankle motor control, ankle robot, hemiparesis, jerk, learning rate, motor adaptation, motor learning, movement smoothness, neurorehabilitation, stroke.

\footnotetext{
Abbreviations: $\mathrm{AL}=$ associative motor learning; $\mathrm{AROM}=$ active range of motion; $\mathrm{CL}=$ cognitive learning; $\mathrm{DF}=$ dorsiflexion; DOF = degree of freedom; GRECC = Geriatric Research, Education, and Clinical Center; HP = hemiparetic; INV/EV = inversion-eversion; LL = lower limb; MA = motor adaptation; MAS = Modified Ashworth Scale; ML = motor learning; MMT = Manual Muscle Test; NIHSS = National Institutes of Health Stroke Scale; PF = plantar flexion; ROM = range of motion; STMA = short-term motor adaptation; STML = shortterm motor learning; UL = upper limb; VA = Department of Veterans Affairs; VAMC = VA medical center.

* Address all correspondence to Dr. Anindo Roy, Department of Neurology, University of Maryland School of Medicine, 10 N Greene St, BC-101, Baltimore, MD 21201; 410605-7000, ext 4321; fax: 410-605-7913.

Email: Aroy@som.umaryland.edu

DOI:10.1682/JRRD.2010.04.0078
} 


\section{INTRODUCTION}

With close to 800,000 new cases reported each year, including 15,000 veterans [1], stroke is the leading cause of chronic disability in the United States [2], with increased prevalence expected from the aging baby boomer generation. The associated healthcare costs are projected to exceed $\$ 2.2$ trillion by 2050 [2]. Persistent lower limb (LL) weakness from hemiparesis often results in functional motor deficits for many with stroke [3-4]. Hemiparetic (HP) gait, compounded with balance deficits, limits mobility and increases fall risk, with nearly 70 percent suffering fall-related injuries in the first year poststroke [5-7]. These deficits also lead to significant limitations in performing basic activities of daily living, which negatively affect these individuals' ability to participate in community life.

Acquisition of LL motor skill is critical to restoration of motor function and implies attaining a level of performance in a given task that is only achievable through practice, but this process can take weeks to months, and proficiency can diminish over time in the absence of continued practice. Thus, strategies that assess and can enhance short-term skill acquisition or retention are of great scientific and practical interest to the motor learning (ML) and rehabilitation community. Prior studies have investigated motor adaptation (MA) and ML under different conditions, e.g., walking on a split-belt treadmill, in both nondisabled subjects [8-10] and those with cerebellar damage [11]. These studies show that the uninjured or injured brain can adapt and store new locomotor patterns after short bouts of training; however, whether this is true at the HP ankle is not known. The HP ankle is especially worthy of investigation because it contributes to slow speed [12-15], asymmetric joint kinematics and kinetics [12-15], prolonged stance duration on the paretic side [12-15], and an impaired ability to coordinate muscles during locomotion [16-18]. Despite this, to our knowledge, no studies have investigated short-term MA (STMA) and short-term ML (STML) at the HP ankle.

At the Baltimore Department of Veterans Affairs (VA), we have developed a modular impedance-controlled ankle robot (anklebot) to deliver task-specific locomotor training aimed at improving paretic ankle contributions to walking and balance function after stroke [19]. The two degree-of-freedom (DOF) anklebot is capable of actuating the ankle joint in dorsiflexion-plantar flexion (DF/PF) as well as inversion-eversion (INV/EV) ranges of motion (ROMs) and is designed to operate in multiple therapeutic settings. The anklebot is interfaced with computer games "played" by moving the ankle in DF/PF and INV/EV ROMs. Because of the impedance control, the anklebot can be programmed in an interactive mode that "assists as needed"; i.e., it encourages volitional movement and provides assistance when users are unable to perform the prescribed movement.

Here we report findings from a single session of interactive anklebot training in a seated position in a sample of individuals with chronic hemiparesis. Evidence from upper limb (UL) studies suggests that physical interaction or interactive training produces the greatest gains in recovery of motor function compared with purely passive or resistive modes [20]; we chose to use a robot for training in part because robots lend themselves to application of ML principles that are now widely accepted as a modern basis for effective rehabilitation. Compared with a human therapist, robots allow for massed repetitive practice of affected limbs and can provide therapists with a variety of practice conditions that can be tailored to the needs of individual patients. The training paradigm in this study included a novel computer video interface motivated by prior studies with UL robot-aided rehabilitation; i.e., the visual interface was used as a customizable training environment in which patients could be challenged relative to their level of deficit. Our purpose was to investigate the short-term effects of paretic ankle training on ankle MA and ML and to assess whether robotic, performance-based, progressive challenge has the potential to be a flexible ML tool poststroke. Specifically, we asked whether a single session of anklebot visuomotor training could evoke positive changes in paretic ankle motor control with robust retention at 48 hours follow-up. We hypothesized that a single session of seated interactive anklebot training with a simple visuomotor targeting task would show greater gains in selected metrics of paretic ankle motor control in people with stroke compared with a similar cohort of ageand sex-matched nondisabled control subjects.

\section{METHODS}

\section{Participants}

A convenience sample of seven $(n=7)$ nondisabled volunteers and an equal number of participants with 
chronic stroke were recruited to participate in a training program with the anklebot. Inclusion criteria for stroke participants included (1) index stroke $>6$ months prior in women or men aged 18 to 85 years, (2) residual HP gait deficits, (3) completion of all conventional physical therapy, (4) adequate language and neurocognitive function to participate in testing and training, and (5) presence of trace DF (Manual Muscle Test [MMT] score $\geq 2$ of 5 ; gravity neutral). Exclusion criteria included (1) fixed or painful contractures at the paretic ankle that could impede participation; (2) marked increase in muscle tone (Modified Ashworth Scale [MAS] >2 at paretic ankle); (3) cerebellar ataxia; (4) sensory deficits defined by loss of proprioception at the great toe $(\geq 4 \mathrm{~mm}$ at distal interphalangeal joint by manual test) and/or hemisensory neglect upon neurological examination; or (5) neurological history of dementia (Mini-Mental Status Examination $\leq 23$ for $>9$ th grade education), receptive or global aphasia with inability to follow two-step commands, nonstroke neurological disorder that could alter ankle motor control, or untreated major depression. The primary exclusion criterion for nondisabled volunteers was the presence of ankle injury that limited full ROM.

\section{Apparatus (Anklebot)}

The design and preclinical characterization, including measurement and torque generation capabilities, of the impedance-controlled anklebot (Interactive Motion Technologies, Inc; Watertown, Massachusetts) have been described in detail elsewhere prior to this pilot study [19]. Briefly, the anklebot is a three-DOF wearable exoskeleton that allows normal ankle ROM in all three DOF of the foot relative to the shank but actuates only DF/PF and INV/EV, with up to $17 \mathrm{Nm}$ of ankle torque. Low static friction and inertia make the device highly backdriveable.* The anklebot can estimate ankle kinematics and kinetics with a very high degree of precision [19] and can be used as an effec-

\footnotetext{
* Backdriveability is a feature in some robots that allows an elderly or impaired individual with diminished limb function to easily move the robot endpoint and allows the robot to interact stably and safely with the environment (individual) at all times. Backdriveability is achieved when the forces produced at the robot endpoint as a result of endpoint motions are low, so that they do not impose a force or position on the individual; i.e., the robot "easily gets out of the way." A backdriveable robot thus allows individuals to train their own movements. Backdriveability is essential in keeping the individuals engaged in an interactive task and enables them to observe their attempts at motion.
}

tive clinical measurement instrument in estimating paretic ankle stiffness [21-22]. Walking with the unpowered anklebot as an added mass on the paretic leg does not interfere or significantly alter gait kinematics [23].

\section{Anklebot Setup}

The anklebot's proximal attachment is mounted anterior to a top-of-the-line orthopedic knee brace (Townsend Design; Bakersfield, California) that is retrofitted with two bicycle-type, rapid connect-disconnect quick-release locks, while the distal attachment is secured to a modified orthopedic shoe via quick connectors [19]. Pads provide additional protection where subjects indicate sensitivity to pressure, and a snug fit in the shoe is aided with the use of foam insoles and socks as required. Excluding the time required to determine the patient's knee brace and shoe sizes, the donning process requires no more than 2 minutes from a single clinician.

\section{Anklebot Control System}

The anklebot controller is a basic impedance controller with programmable reference position, programmable proportional gain (approximating a controllable torsional stiffness), and programmable derivative gain (approximating a controllable torsional damping in parallel with the stiffness) as shown in Figure 1(a). The impedance controller enables "assist-as-needed" delivery of torques at the paretic ankle based on the error between the target location (or a reference) and the proximity of the subject's ankle to the target (or a reference), as well as the robotic torsional stiffness and damping [19]. This control approach allows subjects to reach targets unassisted and automatically tracks their performance; however, if subjects cannot move their ankles to reach a target in time, the robot provides assistive ankle torques.

\section{Procedures}

\section{Pretraining}

After providing informed consent, all subjects underwent routine medical evaluations in the Baltimore VA Geriatric Research, Education, and Clinical Center (GRECC) Assessment Clinic that included passive ROM and ankle active ROM (AROM) measured from a neutral $90^{\circ}$ position with a goniometer (Jamar EZ-Read, Jamar, Inc; Clifton, New Jersey) with their ankle freely suspended under the influence of gravity. Subjects with stroke were also administered the National Institutes of 


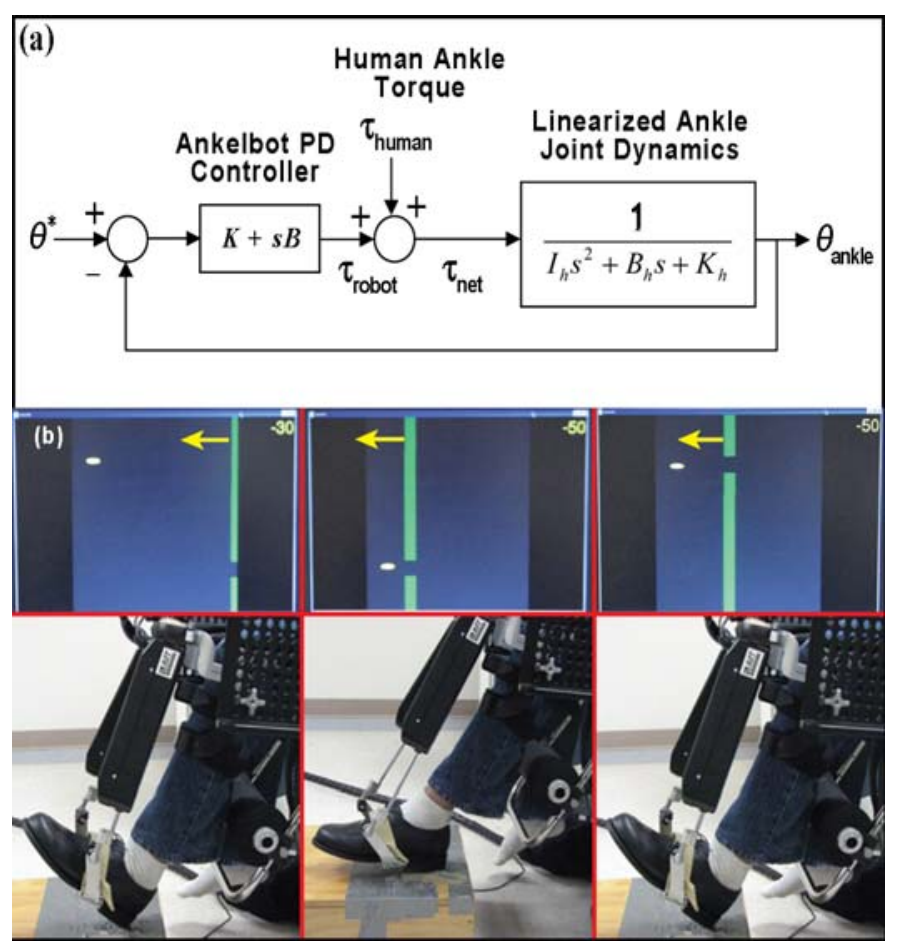

Figure 1.

(a) Schematic representation of anklebot control system (Roy A, Krebs HI, Williams DJ, Bever CT, Forrester LW, Macko RF, Hogan N. Robot-aided neurorehabilitation: A novel robot for ankle rehabilitation. IEEE Trans Robotics. 2009;25(3):569-82. DOI:10.1109/ TRO.2009.2019783). In figure, $\theta$ is ankle angle in dorsiflexion/plantar flexion or inversion-eversion as measured from anatomical neutral; $\theta^{*}$ is reference angle to proportional-derivative (PD) servo, e.g., target location; $I_{h}$ is moment of inertia of human ankle; $K$ and $K_{h}$ are torsional robot and human ankle stiffness, respectively; $B$ and $B_{h}$ are torsional robot and human ankle viscous damping, respectively; and $\tau_{\text {robot }}$, $\tau_{\text {human }}$, and $\tau_{\text {net }}$ are robot, human, and net torques, respectively. (b) Photograph of anklebot being used during seated training with video feedback (adapted with permission from Forrester LF, Roy A, Krebs HI, Macko RF. Ankle training with a robotic device improves hemiparetic gait after a stroke. Neurorehabil Neural Repair. 2011;25:369-77). Arrows denote motion of vertical gates that serve as targets for anklebot-controlled cursor. Knee brace is mounted to fixed plate that supports anklebot and restricts knee and hip motions, effectively isolating ankle to move freely in either dorsiflexion/plantar flexion or inversion-eversion, while heel maintains contact with base to provide a pivot for foot.

Health Stroke Scale (NIHSS) examination and the MAS and MMT (only in DF/PF); with the exception of the NIHSS, all preclinical tests were repeated three times by the same evaluator to obtain test-retest reliability.

Subjects sat in an adjustable chair wearing the anklebot on their paretic/dominant leg with the knee brace secured to a mounting plate for support and immobilization of the knee (Figure 1(b)). A seat belt was secured around the pelvis to further limit proximal hip and thigh motion. The paretic/dominant leg rested at approximately $45^{\circ}$ on a cushioned support with the heel placed on a base to provide a pivot point, thus isolating the foot so it could move freely about the ankle. After the setup, subjects were introduced to the video racing game that constituted the primary activity in subsequent training.

\section{Visuomotor Task}

The video game consisted of targets that approached across the display screen at different vertical levels. As each target appeared, subjects were required to either dorsiflex or plantarflex their paretic/dominant ankle to move a robot-controlled cursor up or down on a display screen to pass through targets ${ }^{*}$ (Figure 1(b)). Successful or unsuccessful clearance scored \pm 10 points per target, respectively, with cumulative progress indicated on the screen in real time. Target locations were predetermined for each individual and standardized at \pm 80 and \pm 40 percent of their AROM in the DF and PF ranges, respectively. These four target locations appeared in a top (DF)-bottom (PF)intermediate (DF)-intermediate (PF) sequence; this was done to provide an equal number of targets at each location and balance the number of shifts between the highlow targets and the number of partial range shifts.

\section{Training Protocol}

The training session lasted an hour, and subjects returned for a follow-up visit 48 hours later. During training, each subject underwent a standard series of 8 blocked trials for a total of 560 targeted ankle movements. The number of targets per block was determined on the basis of prior psychophysical experiments with the UL in nondisabled subjects [24-26]. The first (pre) and the last (post) blocks consisted of 40 targets each in which the robot generated no perturbation (assistance) but only recorded the ankle kinematics ("unassisted trials"). The 6 intermediate blocks each consisted of 80 targets with varying virtual mechanical environments; i.e., robotic assistance in these blocks followed an "easy-to-difficult” sequence (Figure 2), with stronger assistance during the first two blocks ( $K=$ $100 \mathrm{Nm} / \mathrm{rad}$ ), reduced assistance in the next two blocks ( $K=50 \mathrm{Nm} / \mathrm{rad}$ ), and minimum assistance during the last two blocks (at $K=25 \mathrm{Nm} / \mathrm{rad}$ ). This was done to maintain enthusiasm and avoid frustration, especially among

\footnotetext{
*Note that the joint coordinates were different from the visual coordinates in order to compensate for the rectangularity of the monitor.
} 


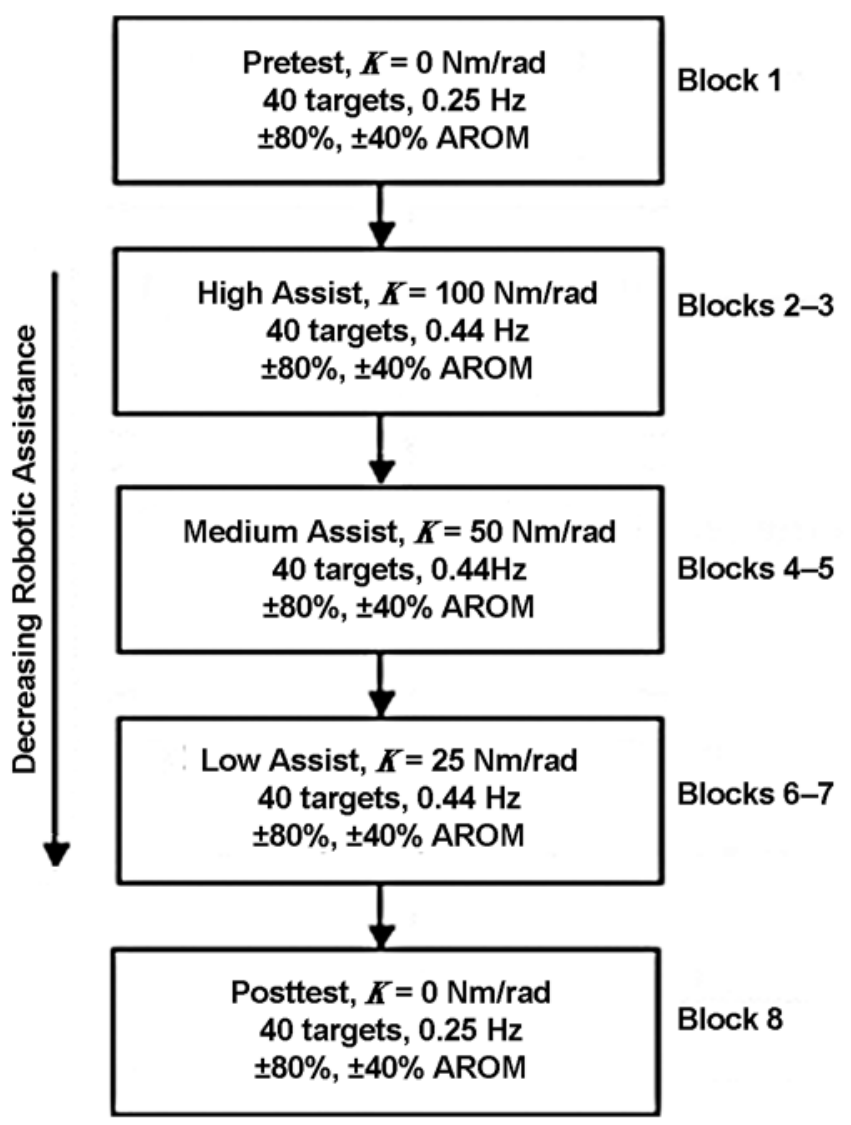

Figure 2.

"Easy-to-difficult" progression algorithm for robotic assistance used in anklebot interactive training. Target locations are set to match each subject's baseline ankle active range of motion (AROM). Frequency (in hertz) in block refers to frequency of target appearance in that block.

subjects with stroke, while providing a degree of challenge specific to deficit severity. Between blocks, the subjects rested for 2 minutes, with additional or longer rest periods provided upon request.

\section{Outcome Evaluation}

\section{Data Analysis}

We calculated robot-based metrics from kinematic data recorded by the robot during pre-post training unassisted trials and the unassisted trial at the 48-hour retest [27-29]; each trial consisted of 40 point-to-point movements. ${ }^{*}$ These included the number of successful passages within a trial, peak speed, mean speed, normalized jerk, and the number of peaks in a speed profile ("peaks metric"). The number of successful passages was directly related to the cumulative score displayed on the screen. We obtained movement velocity and acceleration from the first and second forward-differentiation of position using the MATLAB "Diff” function (The MathWorks, Inc; Natick, Massachusetts). Speed profiles were derived by taking the absolute value of the velocity profiles and then used to calculate mean speed, peak speed, and the peaks metric. Acceleration profiles were forward-differentiated to calculate average jerk and then normalized to peak speed so as to not confound them with changes in overall movement speed. Note that the jerk and peaks metrics are measures of nonsmoothness, so a lower value represents smoother movement and vice versa. In order to account for initial acclimatization, we did not include the first and last four movements in the calculations.

\section{Operational Definitions of STMA, STML, and Treatment Response}

For purposes of this study, STMA refers to the relative change in a performance metric during the unassisted trials at the start versus the end of training (see "Training Protocol"). STML in a metric was considered to be the maintenance of adaptation or retention of performance in that metric over a short period of time (in this case 48 hours), assessed by statistically comparing its values during the unassisted trials at the end of training versus the 48-hour retest. Treatment response refers to the relative change in a metric during the unassisted trials at the 48-hour retest versus the start of training. These definitions are illustrated for exemplar data in Figure 3.

\section{Learning Rates}

To determine differences in learning rates of different motor skills, we used a power law of practice [25-26] that assumes motor performance to be an exponential function of trial number, so that the rate of learning is computed as the coefficient of the exponent, i.e., the slope of the metric with both the metric and the target number plotted on logarithmic scales:

$$
Y=N^{a} \text { or } \log _{10}(Y)=a \times \log _{10}(N),
$$

where $Y$ is the motor control metric, $N$ is the target number, and $a$ is the learning rate. In this study, we computed

\footnotetext{
*A movement was considered to begin when the speed first became greater than 2 percent of the peak speed and was considered to end after the speed dropped and remained below 2 percent of the peak speed.
} 


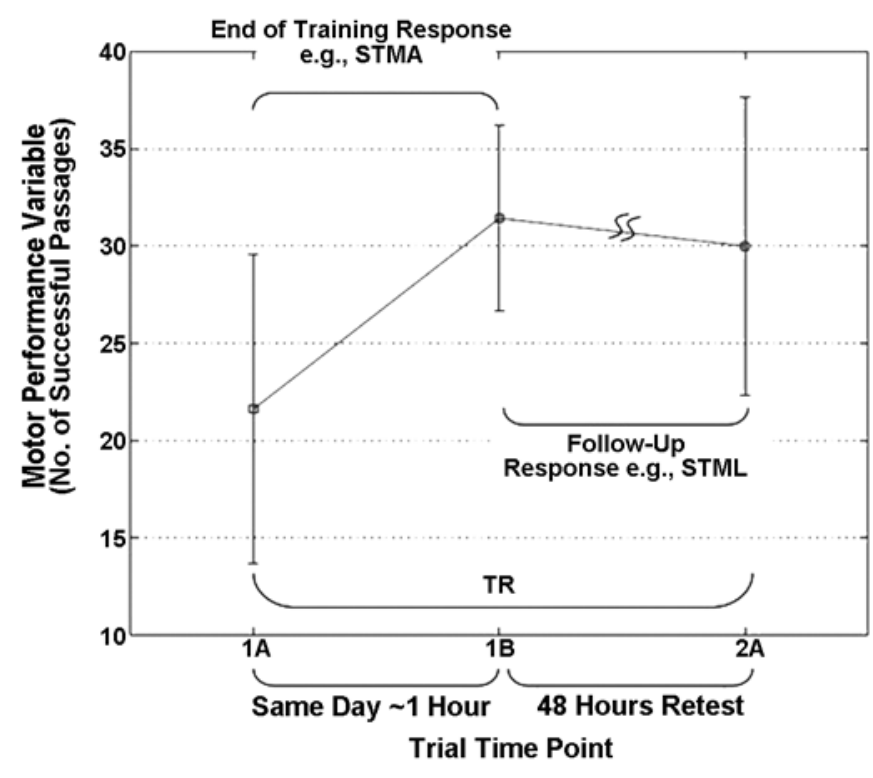

Figure 3.

Exemplar data showing temporal progression of motor control metric (number of successful passages) to illustrate time points used to define short-term motor adaptation (STMA), short-term motor learning (STML), and treatment response (TR). $1 \mathrm{~A}$ and $1 \mathrm{~B}$ refer to pre and post unassisted trials on first day of training, respectively; 2A refers to unassisted trial at 48-hour follow-up.

the learning rate for only one key descriptive measure of motor performance, i.e., the inverse of normalized jerk in the first unassisted trial. Because jerk directly characterizes nonsmoothness, we chose its inverse to characterize movement smoothness; hence, a negative value of learning rate for inverse of movement smoothness would indicate that learning occurred in this variable at a rate equal to its absolute value.

\section{Statistical Analyses}

All outcome variables of interest were tested for changes at three time points: ${ }^{*}$ between the first (" $1 \mathrm{~A}$ ") and last ("1B") unassisted trials and retest at 48 hours ("2A") (Figure 3). For each variable, the subject means for each trial were independently tested for normality of distribution using the Kolmogorov-Smirnov one-sample test. We used the nonparametric Wilcoxon signed rank test to compare pre-post follow-up to detect significant

\footnotetext{
*Here, " $1 \mathrm{~A}$ " and " $1 \mathrm{~B}$ " refer to pre and post unassisted trials on the day of training, respectively; " $2 \mathrm{~A}$ " refers to the unassisted trial at followup (48 hours).
}

differences across the three time points. The significance level was set at $p<0.05$.

\section{RESULTS}

Subject demographics are summarized in Table 1. Each group consisted of seven subjects of the same sex (5 women and 2 men) and paretic/dominant leg (3 right and 4 left) distributions. The stroke group consisted of chronic HP stroke patients who had had their first unilateral infarct from 29 to 146 months ago (77.5 \pm 36.5 months; all data presented as mean \pm standard deviation, where applicable) and were stable in their motor function. Stroke subjects were between 43 and 75 years of age (63.7 \pm 10.5 years), had persistent LL hemiparesis (3 left and 4 right paretic), had impaired AROM in DF $\left(-7.1^{\circ} \pm 11.7^{\circ}\right)$ but normal ranges in $\mathrm{PF}\left(36^{\circ} \pm 10^{\circ}\right)$, had at least Grade 2 or higher on the MMT (77.8 $\pm 47.8 \mathrm{~N}$ paretic dorsiflexor strength), and were mild to moderately spastic (DF: $0-2$, PF: $0-1+$ [MAS]). Five of the seven stroke subjects relied on some type of assistive device for ambulation, e.g., single-point

Table 1.

Demographics of stroke participants $(n=7)$ and nondisabled control subjects $(n=7)$.

\begin{tabular}{|c|c|c|}
\hline Attribute & Stroke & Control \\
\hline Age (yr) & $63.7 \pm 10.5$ & $56.5 \pm 7.5$ \\
\hline $\operatorname{Sex}(M / F)$ & $2 \mathrm{M}, 5 \mathrm{~F}$ & $2 \mathrm{M}, 5 \mathrm{~F}$ \\
\hline Paretic/Dominant ${ }^{*}$ Side $(\mathrm{R} / \mathrm{L})$ & $3 \mathrm{R}, 4 \mathrm{~L}$ & $3 \mathrm{R}, 4 \mathrm{~L}$ \\
\hline Active ROM: DF $\left(^{\circ}\right)$ & $-7.1 \pm 11.7$ & $11.7 \pm 4.1$ \\
\hline Active ROM: $\mathrm{PF}\left({ }^{\circ}\right)$ & $36 \pm 10$ & $47.3 \pm 6.4$ \\
\hline Time Poststroke $^{\dagger}$ (mo) & $77.5 \pm 36.5$ & - \\
\hline Assistive Device & 3 AFO, 3 SPC, 1 QC & - \\
\hline NIH Stroke Scale $\ddagger$ & $0-14$ & - \\
\hline $\mathrm{MMT}^{\S}(\mathrm{N})$ & $77.8 \pm 47.8$ & NT \\
\hline MAS: DF" & $0-2$ & NT \\
\hline MAS: PF & $0-1+$ & NT \\
\hline
\end{tabular}

Note: Nonnumeric data are expressed as distribution. MMT, MAS, and NIH Stroke Scale scores are reported as group range, while sex and side tested are reported as distributions.

*Self-reported dominant side.

${ }^{\dagger}$ Time poststroke reported in number of months after occurrence of first stroke. ${ }^{\ddagger}$ NIH Stroke Scale scores range from 0 (normal) to 42 (most severe).

${ }^{\S}$ MMT performed for paretic dorsiflexor muscles using handheld dynamometer and scores reported are peak value of three trials for each subject (in newtons). "MAS scores range from 0 (no muscle tone) to 4 (limbs rigid in flexion or extension).

AFO = assistive foot orthosis, $\mathrm{DF}=$ dorsiflexion, $\mathrm{F}=$ female, $\mathrm{L}=$ left, $\mathrm{M}=$ male, MAS $=$ Modified Ashworth Scale, MMT $=$ Manual Muscle Test, NIH = National Institutes of Health, $\mathrm{NT}=$ not tested, $\mathrm{PF}=$ plantar flexion, $\mathrm{QC}=$ quad-piece cane, $\mathrm{R}$ = right, $\mathrm{ROM}$ = range of motion, $\mathrm{SPC}$ = single-piece cane. 
cane. The age-matched control group consisted of nondisabled subjects who were between 50 and 64 years of age (56.5 \pm 7.5 years) and had normal AROM in both directions (DF: $11.7 \pm 4.1^{\circ}$, PF: $47.3 \pm 6.4^{\circ}$ ). Figure 4 shows two sets of traces of 40 targeted movements during an unassisted trial, one made by a representative stroke subject and the other by a nondisabled subject both before and after training. In every case, all trial distributions of the group data proved to be non-normally distributed, hence, requiring the use of nonparametric statistics.

\section{STMA and Magnitude of Treatment Response}

The stroke group showed statistically significant gains in all five motor control variables at the end of training (Table 2): normalized jerk (654.1 $\pm 103.3 \mathrm{~s}^{-2}$ to $\left.537.6 \pm 86.7 \mathrm{~s}^{-2}, p=0.001\right)$ and the peaks metric (27.1 \pm 5.8 to $23.7 \pm 4.1, p=0.009$ ) decreased, while mean speed $(4.7 \pm 1.5 \%$ s to $6.5 \pm 2.6 \%$ s, $p=0.007)$, peak speed $(42.8 \pm 9.0 \%$ s to $45.6 \pm 9.4 \% \mathrm{~s}, p=0.03)$, and the number of successful passages (21.6 \pm 8.0 to $31.4 \pm 4.8, p=0.04$ ) increased, indicating STMA in these variables. In contrast, the nondisabled group did not significantly improve in any of the motor control variables at the end of train-
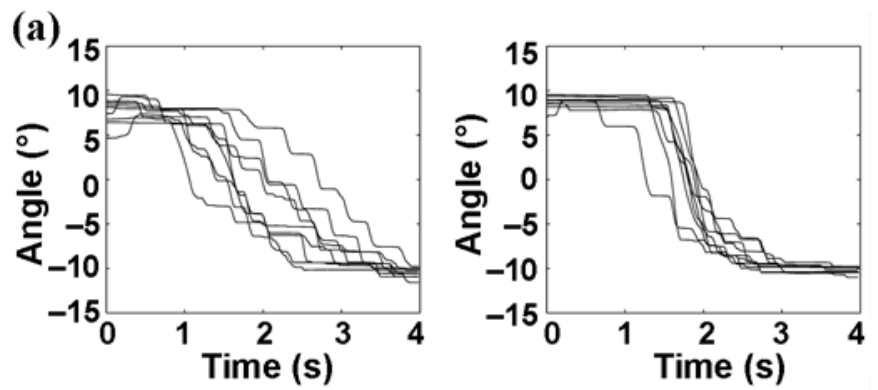

(b)
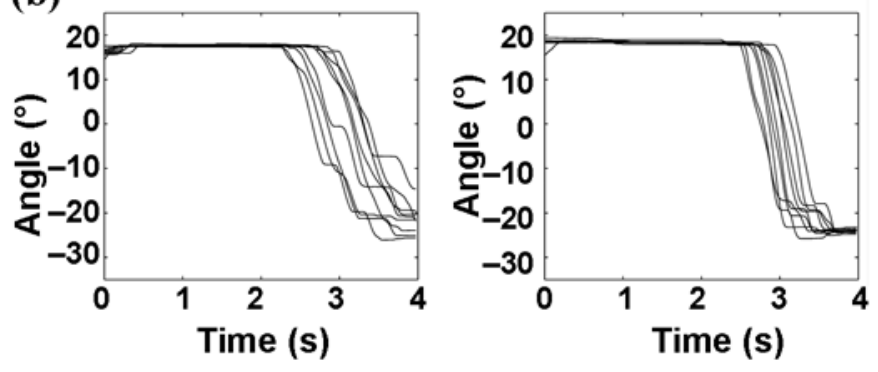

Figure 4.

Ankle angle versus time trace during set of point-to-point movements made by (a) typical stroke and (b) nondisabled control subject during an unassisted (40-target) trial at start (left panel) and end of training (right panel). Both subjects were required to plantar flex in order to successfully maneuver through gate. ing, with the exception of one variable, i.e., number of successful passages ( $32.3 \pm 7.5$ to $36.5 \pm 6.4, p=0.006$ ).

When compared with the 48-hour retest, the magnitude of the TR in those with stroke also showed improvements in all five variables (Table 2): number of successful passages ( $21.6 \pm 8.0$ to $30.0 \pm 7.7, p=0.01)$, mean ( $4.7 \pm$ $1.5 \%$ s to $6.5 \pm 3.0 \%$ s, $p=0.03)$ and peak speeds ( $42.8 \pm$ $9.0 \%$ to $47.1 \pm 11.3 \%$ s, $p=0.05$ ), normalized jerk (548.5 $\pm 99.4 \mathrm{~s}^{-2}$ to $\left.654.1 \pm 103.3 \mathrm{~s}^{-2}, p=0.03\right)$, and the peaks metric ( $27.1 \pm 5.8$ to $22.9 \pm 4.2, p=0.006)$. The control group, however, did not show increases in TR in any of the motor performance metrics (Figure 5).

\section{Performance Retention after 48 Hours}

Importantly, the performance gains in all motor control metrics made by both groups at the end of training were retained at 48 hours as evidenced by the lack of statistically significant differences between the two time points. Subjects retained their movement accuracy: number of successful passages (stroke: $31.4 \pm 4.8$ to $30.0 \pm 7.7$, $p=0.61$; control: $36.5 \pm 6.4$ to $35.0 \pm 9.3, p=0.4$ ), movement speed (mean) (stroke: $6.5 \pm 2.6 \%$ s to $6.5 \pm 3.0 \%$, $p=0.9$; control: $18.1 \pm 4.3 \%$ s to $17.9 \pm 2.6 \%$ s, $p=0.9$ ), peak speed (stroke: $45.6 \pm 9.4 \%$ s to $47.1 \pm 11.3 \%$ s, $p=$ 0.36; control: $93.5 \pm 17.2 \%$ s to $94.0 \pm 13.9 \%$ s, $p=0.3$ ), movement smoothness (normalized jerk) (stroke: $537.6 \pm$ $86.7 \mathrm{~s}^{-2}$ to $548.5 \pm 99.4 \mathrm{~s}^{-2}, p=0.7$; control: $404.1 \pm$ $143.3 \mathrm{~s}^{-2}$ to $416.7 \pm 82.1 \mathrm{~s}^{-2}, p=0.8$ ), and the peaks metric (stroke: $23.7 \pm 4.1$ to $22.9 \pm 4.2, p=0.3$; control: $35.8 \pm$ 9.0 to $35.8 \pm 4.6, p>0.99$ ). This robust postadaptation retention was present in all subjects in both groups and may suggest some form of STML (see "Discussion”).

\section{Rate of Learning of Movement Smoothness}

The learning rates for inverse normalized jerk were negative for both groups, with higher magnitudes and intersubject variability for the nondisabled group $(-2.1 \pm$ $1.6)$ than for stroke subjects $(-1.86 \pm 0.07)$. Negative values indicated that subjects in both groups began to make smoother movements with the passage of time during the first unassisted trial.

\section{DISCUSSION}

\section{Summary}

The primary findings of this study were that a single session of seated visuomotor anklebot training caused significant changes in five key descriptors of motor control 
JRRD, Volume 48, Number 4, 2011

Table 2.

Summary of gains in motor performance variables during short-term motor adaptation (STMA) and short-term motor learning (STML) and the magnitude of treatment response (TR) for stroke and age-matched control subjects.

\begin{tabular}{|c|c|c|c|c|c|}
\hline \multirow{2}{*}{ Behavior } & \multirow{2}{*}{$\begin{array}{c}\text { Accuracy: } \\
\text { No. of Successes }\end{array}$} & \multicolumn{2}{|c|}{ Smoothness } & \multicolumn{2}{|c|}{ Speed (\%/s) } \\
\hline & & Normalized Jerk $\left(\mathrm{s}^{-2}\right)$ & No. of Peaks & Mean & Peak \\
\hline \multicolumn{6}{|l|}{ Stroke Group } \\
\hline STMA & 45.4 & -17.8 & -12.6 & 38.9 & 6.4 \\
\hline STML & -4.5 & 2.0 & -3.6 & -0.2 & 3.4 \\
\hline TR & 38.8 & -16.1 & -15.8 & 38.6 & $9.9^{*}$ \\
\hline \multicolumn{6}{|l|}{ Control Group } \\
\hline STML & -4.1 & 3.1 & 0 & -1.1 & 0.5 \\
\hline TR & 8.4 & -22.4 & -16.7 & 21.8 & 15.6 \\
\hline
\end{tabular}
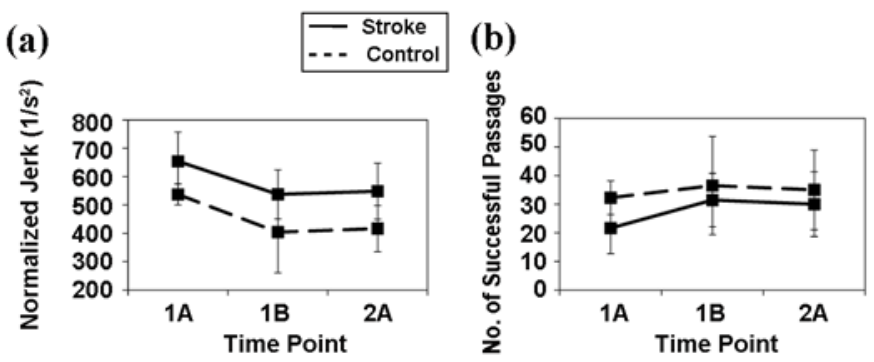

(c)

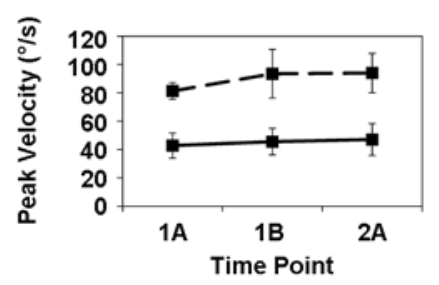

(d)

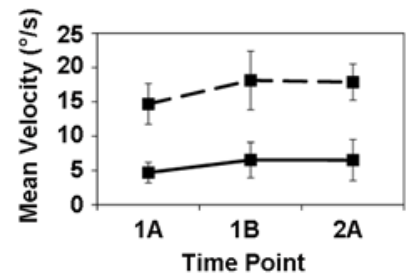

Figure 5.

Profiles of motor control metrics (a) normalized jerk, (b) number of successful passages, (c) peak speed, and (d) mean speed at three time points, i.e., first and last unassisted trials on day of training and unassisted trial at 48 hours. $1 \mathrm{~A}$ and $1 \mathrm{~B}$ refer to pre and post unassisted trials on first day of training, respectively; 2A refers to unassisted trial at 48-hour follow-up.

at the HP ankle, including movement speed (peak and mean speed), smoothness (normalized jerk and peaks metric), and accuracy (number of successful targeted movements) and that all performance gains were maintained 48 hours later. In contrast, age-matched nondisabled individuals did not show improvement in any but one motor performance variable. Moreover, negative values of learning rate of the inverse of normalized jerk during initial task exposure indicated that both nondisabled and stroke subjects began to make smoother movements early during the training session, with nondisabled individuals learning the task sooner than those with stroke. This trend continued over the course of training, with subjects in both groups becoming increasingly proficient at making more successful, faster, and smoother movements, but STMA in these motor skills as indicated by statistical differences in motor performance before and after training occurred only at the HP ankle.

\section{Methodological Considerations and Comparison to Other Motor Learning Paradigms}

\section{Paradigm and Motor Control Metrics}

The experimental paradigm used here presented subjects with a "virtual mechanical environment" that required them to relearn motor synergies to accurately acquire targets with efficient, smooth ankle movements by moving the robot end-effector from its initial position toward a target. This visually evoked and guided training task is similar to that used in multiple ML studies for the UL, including a recent multisite VA cooperative trial [24-26,30]. The selection of our motor control measures was based in part on performance indices used and validated in studies for arm reaching investigating ML in human subjects within a virtual mechanical environment generated by a perturbation force field [24-26,31-33]. For example, normalized jerk and peak and mean speed have been used to quantify motor performance in nondisabled and neurologically impaired individuals [2729,34]. However, unlike this study, none of those studies 
examined the immediate profile of early STMA and STML in arm motor control.

\section{Comparison to Other Lower-Limb Paradigms}

This study is by no means the first to investigate MA and ML at the LL. Several studies have explored this topic but mostly in the context of gait [8-11]. However, those studies differ from ours in that they investigated MA and ML in the leg as a whole and used spatio-temporal gait parameters [9], joint angle-based gait symmetry [10], or muscle electromyogram $[8,10]$ as metrics, whereas here we focused on studying STMA and STML at the HP ankle using position and torque information. Moreover, those studies used nondisabled subject volunteers [8-10] or subjects with cerebellar damage [11] but not stroke. Also, none of the authors, except Kao and Ferris [8], investigated short-term (e.g., 48 hours) postadaptation aftereffects (retention), thereby limiting their scope in examining ML at the LL.

It is clear from comparisons to prior studies that investigation of STMA/STML at the HP ankle is a significantly neglected topic in motor neuroscience. To our knowledge, only one study's experimental protocol affords limited comparison to our study. Perez et al. investigated short-term changes in ankle motor control under similar experimental conditions; i.e., subjects were instructed to make a cursor follow a series of target lines on a computer screen by performing voluntary ankle DF/ PF movements for a period of 32 minutes [35]. They showed that a single bout of motor skill ankle training improved ankle motor performance as indexed by increased cortical excitability to the tibialis anterior. This was reflected by a significant increase in the recruitment curves obtained using transcranial magnetic stimulation after 32 minutes, suggesting that short-term skill motor training increases motor cortical excitability. They concluded that similar to hand motor skill training, plastic changes may also be observed for the leg motor area and may be related to the degree of difficulty in the motor task. Despite these similarities to our study, their investigation was limited to nondisabled subjects. Hence, we believe that this is the first time that STMA, and possibly STML, is being reported for the HP ankle following a single session of interactive robot training.

\section{Evidence of STMA at Hemiparetic Ankle}

STMA was characterized by improvements in ankle motor control at the HP but not the nondisabled ankle.
Faster, smoother, and more accurate targeted movements, as indicated by higher angular speeds, lower jerk, and higher number of successful passages through the targets before and after training, characterized this behavior. Among nondisabled subjects, however, this behavior was absent in all but one metric. This could be due to a ceiling effect in the first unassisted trial itself, thereby minimizing the effect of training. In stroke subjects, however, improvements in motor performance suggest a more efficient voluntary control of ankle musculature.

\section{How Fast Did Skill Acquisition Occur During Initial Exposure?}

Negative learning rates with magnitudes $>1$ for inverse jerk in both groups indicated that subjects rapidly acquired the ability to make smoother movements during initial exposure in the first unassisted trial, with nondisabled subjects acquiring the ability faster. This could be in part due to the task difficulty, i.e., location of targets scaled to AROM in DF/PF, real-time performance feedback provided to sustain motivation, and a deterministic sequence of targets that alternated between DF and PF.

The magnitude of the average learning rate in each group was higher than those previously reported in the literature, for both neurological (Krebs et al. [36]) and nondisabled [26] subjects. For example, Krebs et al. studied learning deficits in subjects with Parkinson disease and in nondisabled age-matched controls using a robotic device to generate conservative force fields that disturbed the subjects' arm movements, thereby generating a "virtual mechanical environment" that subjects learned to manipulate to perform a goal-oriented visual task [36]. Similar to this study, they found that learning rates for multiple measures of movement smoothness were negative, but lower by one order of magnitude $\left(\sim 10^{-2}\right)$ compared with the present study $\left(\sim 10^{-1}\right)$. Differences in paradigm (fixed vs scaled and random vs deterministic target locations), disease population (Parkinson vs chronic-stage stroke), limb or joint trained (UL vs LL), and age may have contributed to the difference in magnitudes of learning rates. In Krebs et al.'s study, subjects in both groups (mean age of Parkinson $\sim 72$ years, nondisabled $\sim 75$ years) [36] were significantly older than those who participated in this study (mean age of stroke 63 years, nondisabled $\sim 56$ years). In light of this result, one might wonder and speculate about the benefits of scaling task difficulty and the effect of aging on short-term ankle ML and what surrogate markers could be anticipated from such a research. 


\section{Could Retention of Motor Performance at 48 Hours Be Evidence of STML?}

Studies have suggested that improvements in movement smoothness, as reported here, are the result of a learned, coordinative process rather than inherently due to the structure of the neuromuscular system [37-38]. The robust postadaptation aftereffects (retention) in all the motor performance gains at 48 hours may suggest some form of rapid, short-term ankle ML, but its exact nature or underlying mechanism (e.g., blending of discrete submovements as recovery occurs [39]) cannot be ascertained unless a longer time window is explored. It may be cognitive learning $(\mathrm{CL})^{*}$ (as opposed to implicit or procedural learning), which is the first stage of learning in Fitts and Posner's three-stage ML model [40]. During this stage, subjects successfully formed the overall concept of the task by gaining information through their visual and proprioceptive senses, followed by carryover at 48 hours retest. However, because our task consisted of a predictable sequence of targets ("up"/DF-“down”/PF"up"/DF), it is possible that this form of ML could be associative motor learning (AL), which is the second stage in Fitts and Posner's model. During AL, subjects learn to perform a task with greater efficiency and accuracy, moving toward overall refinement of skill after having cognitively learned the task [40]. Therefore, our interactive training could have evoked STML at the paretic ankle with features that are characteristic of both CL and AL, as in Adam's two-stage ML model [41]. These trends are also consistent with Gentile's two-stage model [34], in which subjects first accomplish "getting the idea of the movement"; i.e., the learner understands how the movement should be organized to accomplish the task, followed by the "fixation" stage in which the subject learns to make consistent movements or repeat actions within the presented environment.

\section{Relationship of Task to Locomotor Function}

An important question is whether and how the training task used in this study relates to locomotor function. Ankle training in a seated position in response to visual stimuli does not appear to be explicitly related to training whole-body (i.e., gait) function; however, because the heel was "pivoted" on a platform during execution of the task, PF emulates the initial loading during the stance

\footnotetext{
*Sometimes also referred to as “verbal cognitive learning” in the literature.
}

phase of gait following heel strike, while DF partly ${ }^{\dagger}$ characterizes the initial-to-mid swing phase following toe-off. Therefore, even though the training activity is not task-specific in terms of training locomotor function, we believe that it indirectly trains two critical phases of the gait cycle that require subjects to learn and exercise better PF, i.e., controlled landing following heel-strike and DF control, in order to make successful targeted movements. It is also important to point out that unlike these two phases of the gait cycle, our intervention does not train the ankle plantar flexors for propulsion dynamics during terminal stance; however, we believe that a more efficient motor control of ankle musculature as evidenced in the present study, even in a closed-chain condition, may improve anterior-posterior (A/P) propulsion dynamics if subjects are exposed to additional training sessions. This hypothesis is indeed supported by recent evidence [42] showing that this paradigm may be a valuable adjunct to locomotor therapies in that subjects with stroke who trained 3 times weekly for 6 weeks improved their gait function as indexed by higher unassisted floorwalking speed (20\%), longer duration of paretic singlesupport stance (15\%), and increased A/P positive propulsion during terminal stance ( $18 \%$ in 4 out of 5 subjects).

\section{Study Limitations}

The small sample size limits the generalizability of our findings and requires that they be interpreted with caution; however, this was an initial pilot study to explore the feasibility of using the anklebot as an STML platform and an important first step toward making inroads into this relatively unexplored topic in motor neuroscience. Future studies will be randomized and consist of a control group receiving equivalent nonrobotic or sham therapy.

Although we found STMA and some form of STML in multiple indices of paretic ankle motor control, our paradigm did not assess whether these gains translate into improvements in gait function. Future studies will benchmark these motor performance gains against functional measures, both in the laboratory and in complex, simulated free-living activities. Also, by restricting training to a single visit, we cannot ascertain whether STMA and postadaptation aftereffects continue beyond the 48-hour

\footnotetext{
${ }^{\dagger}$ We say partly because unlike actual initial or midswing, here the heel is in contact with the ground during DF.
} 
time period, i.e., whether interactive ankle training has an additive effect on paretic ankle motor control across additional sessions and whether these sessions could help determine the underlying nature of ML.

\section{CONCLUSIONS}

We presented pilot findings from a study that uses a novel impedance-controlled anklebot to deliver interactive therapy to subjects with chronic HP stroke. Our findings provide the first-of-its kind evidence that a single session of anklebot training evokes STMA at the HP ankle characterized by significant positive changes in the success, speed, and smoothness of targeted ankle movements that were not observed at the nondisabled ankle. Gains in motor performance were retained on retest at 48 hours, potentially suggesting some type of rapid STML in these measures. We believe that the importance of having a tool such as the anklebot capable of assaying LL ML by providing a customizable and adaptive training environment is substantial, particularly if it relates to locomotion, and this work is an important step in understanding the ability of the HP ankle to learn (or relearn) a motor task. Future studies are warranted to further examine any clinical predictors of motor response and to better understand the nature of ML at the HP ankle.

\section{ACKNOWLEDGMENTS}

\section{Author Contributions:}

Study concept and design: A. Roy, L. W. Forrester, R. F. Macko. Acquisition of data: A. Roy.

Analysis and interpretation of data: A. Roy.

Drafting of manuscript: A. Roy.

Critical revision of manuscript for important intellectual content:

A. Roy, L. W. Forrester.

Obtained funding: L. W. Forrester, R. F. Macko.

Study supervision: R. F. Macko.

Financial Disclosures: The authors have declared that no competing interests exist.

Funding/Support: This material was based on work supported by the VA Rehabilitation Research and Development Service (grant B2294T) and the Baltimore VA Medical Center (VAMC) "Center of Excellence on Task-Oriented Exercise and Robotics in Neurological Diseases” (grant B3688R).

Additional Contributions: We acknowledge the GRECC of the Baltimore VAMC as the site of conduct for the clinical research. Dr. Roy acknowledges Jason Diaz for his help with conducting the experiments. Institutional Review: Recruitment and informed consent procedures were approved by the University of Maryland, Baltimore, Institutional
Review Board and the Baltimore VA Research and Development Committee.

Participant Follow-Up: The authors do not plan to inform participants of the publication of this study. Participants have met with the investigators to discuss the insights from their individual training sessions. Disclaimer Statement: The views expressed by contributors are their own and not necessarily the official policy of the VA.

\section{REFERENCES}

1. QUERI Fact sheets [Internet]. Washington (DC): Department of Veterans Affairs; 2009 [updated 2009 Oct 22]. Available from: http://www.hsrd.research.va.gov/publications/queri factsheets/.

2. Heart disease and stroke statistics [Internet]. Dallas (TX): American Heart Association; 2009. Available from: http:// www.americanheart.org/statistics [2009].

3. Katz RT, Rymer WZ. Spastic hypertonia: Mechanisms and measurement. Arch Phys Med Rehabil. 1989;70(2):144-55. [PMID: 2644919]

4. Young RR. Spasticity: A review. Neurology 1994;44(11 Suppl 9):S12-20. [PMID: 7970006]

5. Macko RF, Smith GV, Dobrovolny CL, Sorkin JD, Goldberg AP, Silver KH. Treadmill training improves fitness reserve in chronic stroke patients. Arch Phys Med Rehabil. 2001;82(7):879-84. [PMID: 11441372]

DOI:10.1053/apmr.2001.23853

6. Silver KH, Macko RF, Forrester LW, Goldberg AP, Smith GV. Effects of aerobic treadmill training on gait velocity, cadence, and gait symmetry in chronic hemiparetic stroke: A preliminary report. Neurorehabil Neural Repair. 2000; 14(1):65-71. [PMID: 11228951] DOI:10.1177/154596830001400108

7. Forster A, Young J. Incidence and consequences of falls due to stroke: A systematic inquiry. BMJ. 1995;311(6997): 83-86. [PMID: 7613406]

8. Kao PC, Ferris DP. Motor adaptation during dorsiflexionassisted walking with a powered orthosis. Gait Posture. 2009; 29(2):230-36. [PMID: 18838269] DOI:10.1016/j.gaitpost.2008.08.014

9. Reisman DS, Block HJ, Bastian AJ. Interlimb coordination during locomotion: What can be adapted and stored? J Neurophysiol. 2005;94(4):2403-15. [PMID: 15958603] DOI:10.1152/jn.00089.2005

10. Cain SM, Gordon KE, Ferris DP. Locomotor adaptation to a powered ankle-foot orthosis depends on control method. J Neuroeng Rehabil. 2007;4:48. [PMID: 18154649] DOI:10.1186/1743-0003-4-48

11. Morton SM, Bastian AJ. Cerebellar contributions to locomotor adaptations during splitbelt treadmill walking. J Neurosci. 2006;26(36):9107-16. [PMID: 16957067] DOI:10.1523/JNEUROSCI.2622-06.2006 
12. Perry J. Gait analysis: Normal and pathological function. Thorofare (NJ): SLACK; 1992.

13. Olney SJ, Richards C. Hemiparetic gait following stroke. Part I: Characteristics. Gait Posture. 1996;4(2):136-48. DOI:10.1016/0966-6362(96)01063-6

14. Carlsöö S, Dahlöf AG, Holm J. Kinetic analysis of the gait in patients with hemiparesis and in patients with intermittent claudication. Scand J Rehabil Med. 1974;6(4):166-79. [PMID: 4458059]

15. Chen G, Patten C, Kothari DH, Zajac FE. Gait differences between individuals with post-stroke hemiparesis and nondisabled controls at matched speeds. Gait Posture. 2005; 22(1):51-56. [PMID: 15996592] DOI:10.1016/j.gaitpost.2004.06.009

16. Higginson JS, Zajac FE, Neptune RR, Kautz SA, Delp SL. Muscle contributions to support during gait in an individual with post-stroke hemiparesis. J Biomech. 2006;39(10): 1769-77. [PMID: 16046223] DOI:10.1016/j.jbiomech.2005.05.032

17. Den Otter AR, Geurts AC, Mulder T, Duysens J. Abnormalities in the temporal patterning of lower extremity muscle activity in hemiparetic gait. Gait Posture. 2007;25(3): 342-52. [PMID: 16750632]

DOI:10.1016/j.gaitpost.2006.04.007

18. Berger W, Horstmann G, Dietz V. Tension development and muscle activation in the leg during gait in spastic hemiparesis: Independence of muscle hypertonia and exaggerated stretch reflexes. J Neurol Neurosurg Psychiatry. 1984;47(9): 1029-33. [PMID: 6090590]

DOI:10.1136/jnnp.47.9.1029

19. Roy A, Krebs HI, Williams DJ, Bever CT, Forrester LW, Macko RF, Hogan N. Robot-aided neurorehabilitation: A novel robot for ankle rehabilitation. IEEE Trans Robotics. 2009;25(3):569-82. DOI:10.1109/TRO.2009.2019783

20. Hogan N, Krebs HI, Rohrer B, Palazzolo JJ, Dipietro L, Fasoli SE, Stein J, Hughes R, Frontera WR, Lynch D, Volpe BT. Motions or muscles? Some behavioral factors underlying robotic assistance of motor recovery. J Rehabil Res Dev. 2006;43(5):605-18. [PMID: 17123202] DOI:10.1682/JRRD.2005.06.0103

21. Roy A, Krebs HI, Patterson SL, Judkins TN, Khanna I, Forrester LW, Macko RF, Hogan N. Measurement of human ankle stiffness using the anklebot. Proceedings of the IEEE 10th International Conference on Rehabilitation Robotics; 2007 Jun 13-15; Noordwijk, the Netherlands. Los Alamitos (CA): IEEE; 2007. p. 356-63.

DOI:10.1109/1CORR.2007.4428450.

22. Roy A, Krebs HI, Bever CT, Forrester LW, Macko RF, Hogan N. Measurement of passive ankle stiffness in subjects with chronic hemiparesis using a novel ankle robot. J Neurophysiol. 2011;105(4). [PMID: 21346215] DOI:10.1152/jn.01014.2010
23. Khanna I, Roy A, Rodgers MM, Krebs HI, Macko RM, Forrester LW. Effects of unilateral robotic limb loading on gait characteristics in subjects with chronic stroke. J Neuroeng Rehabil. 2010;7:23. [PMID: 20492698]

DOI:10.1186/1743-0003-7-23

24. Krebs HI, Brashers-Krug T, Rauch SL. Robot-aided functional imaging. DiGioia AM III, Taylor RH, Kikinis R, Lavallee S, editors. Proceedings of the 2nd International Symposium on Medical Robotics and Computer Assisted Surgery; 1995 Nov 5-7; Baltimore, MD. New York (NY): Wiley; 1995. p. 296-99.

25. Krebs HI, Brashers-Krug T, Rauch SL, Savage CR, Hogan N, Rubin RH, Fischman AJ, Alpert NM. Integration of robotic technology with functional imaging. Neuroimage. 1996;3(1 Suppl 1):S394. DOI:10.1016/S1053-8119(96)80396-X

26. Krebs HI, Brashers-Krug T, Rauch SL, Savage CR, Hogan N, Rubin RH, Fischman AJ, Alpert NM. Robot-aided functional imaging: Application to a motor learning study. Hum Brain Mapp. 1998;6(1):59-72. [PMID: 9673663] DOI:10.1002/(SICI)1097-0193(1998)6:1<59::AIDHBM5>3.0.CO;2-K

27. Brooks VB, Cooke JD, Thomas JS. The continuity of movements. In: Stein RB, Pearson KG, Smith RS, Redford JB, editors. Control of posture and locomotion. New York (NY): Plenum Press; 1973. p. 257-72.

28. Fetters L, Todd J. Quantitative assessment of infant reaching movements. J Mot Behav. 1987;19(2):147-66.

[PMID: 14988056]

29. Kahn LE, Zygman ML, Rymer WZ, Reinkensmeyer DJ. Effect of robot-assisted and unassisted exercise on functional reaching in chronic hemiparesis. Proceedings of the 23rd Annual IEEE Engineering in Medicine and Biology Conference; 2001 Oct 25-28; Istanbul, Turkey. Los Alamitos (CA): IEEE; 2001. p. 1344-47.

30. Lo AC, Guarino PD, Richards LG, Haselkorn JK, Wittenberg GF, Federman DG, Ringer RJ, Wagner TH, Krebs HI, Volpe BT, Bever CT Jr, Bravata DM, Duncan PW, Corn BH, Maffucci AD, Nadeau SE, Conroy SS, Powell JM, Huang GD, Peduzzi P. Robot-assisted therapy for longterm upper-limb impairment after stroke. N Engl J Med. 2010;362(19):1772-83. [PMID: 20400552]

DOI:10.1056/NEJMoa0911341

31. Krebs HI. Robot-aided neuro-rehabilitation and functional imaging [dissertation]. Cambridge (MA): Massachusetts Institute of Technology; 1997.

32. Shadmehr R, Holcomb HH. Neural correlates of motor memory consolidation. Science. 1997;277(5327):821-25.

[PMID: 9242612]

DOI:10.1126/science.277.5327.821

33. Shadmehr R, Mussa-Ivaldi FA. Adaptive representation of dynamics during learning of a motor task. J Neurosci. 1994; 14(5 Pt 2):3208-24. [PMID: 8182467] 
34. Gentile AM. Skill acquisition: Action, movement, and neuromotor processes. In: Carr JH, Shepherd RB, Gordon J, Gentile AM, Held JM, editors. Movement science: Foundations for physical therapy in rehabilitation. 2nd ed. Rockville (MD): Aspen; 2000. p. 111-87.

35. Perez MA, Lungholt BK, Nyborg K, Nielsen JB. Motor skill training induces changes in the excitability of the leg cortical area in healthy humans. Exp Brain Res. 2004; 159(2):197-205. [PMID: 15549279] DOI:10.1007/s00221-004-1947-5

36. Krebs HI, Hogan N, Hening W, Adamovich SV, Poizner H. Procedural motor learning in Parkinson's disease. Exp Brain Res. 2001;141(4):425-37. [PMID: 11810137] DOI:10.1007/s002210100871

37. Von Hofsten C. Structure of early reaching movements: A longitudinal study. J Mot Behav. 1991;23(4):280-92. [PMID: 14766510$]$ DOI:10.1080/00222895.1991.9942039

38. Levin MF. Interjoint coordination during pointing movements is disrupted in spastic hemiparesis. Brain. 1996; 119(Pt 1):281-93. [PMID: 8624689] DOI:10.1093/brain/119.1.281

39. Rohrer B, Fasoli S, Krebs HI, Hughes R, Volpe B, Frontera WR, Stein J, Hogan N. Movement smoothness changes dur- ing stroke recovery. J Neurosci. 2002;22(18):8297-8304. [PMID: 12223584]

40. Fitts PM, Posner MI. Learning and skilled performance in human performance. Belmont (CA): Brock-Cole; 1967.

41. Adams JA. A closed-loop theory of motor learning. J Mot Behav. 1971;3(2):111-49. [PMID: 15155169]

42. Forrester LF, Roy A, Krebs HI, Macko RF. Ankle training with a robotic device improves hemiparetic gait after a stroke. Neurorehabil Neural Repair. 2011;25:369-77.

Submitted for publication May 7, 2010. Accepted in revised form January 31, 2011.

This article and any supplementary material should be cited as follows:

Roy A, Forrester LW, Macko RF. Short-term ankle motor performance with ankle robotics training in chronic hemiparetic stroke. J Rehabil Res Dev. 2011;48(4):417-30.

DOI:10.1682/JRRD.2010.04.0078

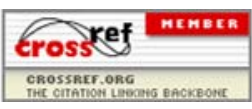


